\title{
CONF- $961202 \ldots$ \\ SMALL ANGLE NEUTRON SCATTERING MODELING OF COPPER-RICH PRECIPITATES IN STEEL
}

Stephen Spooner

Oak Ridge National Laboratory, Oak Ridge, TN 37831, spo@ornl.gov

ABSTRACT
RECEIVED

MAR 061998

The magnetic-to-nuclear scattering intensity ratio observed in the scattering A precipitates in irradiated pressure vessel steels is much smaller than the value of $\mathbf{1 1 . 4}$ expected for a pure copper precipitate in iron. A model for precipitates in pressure vessel steels which matches the observed scattering typically incorporates manganese, nickel, silicon and other elements and it is assumed that the precipitate is non-magnetic. In the present work consideration is given to the effect of composition gradients and ferromagnetic penetration into the precipitate on the small angle scattering cross section for copper-rich clusters as distinquished from conventional precipitates. The calculation is an extension of a scattering model for micelles which consist of shells of varying scattering density. A discrepancy between recent SANS scattering experiments on pressure vessel stells was found to be related to applied magnetic field strength. The assumption of cluster structure and its relation to atom probe FIM findings as well as the effects of insufficient field for magnetic saturation is discussed.

\section{INTRODUCTION}

Small angle neutron scattering (SANS) analysis is used for precipitate characterization of irradiationinduced copper-rich clusters in nuclear reactor pressure steels $[1,2,3]$. These data have contributed to the understanding the effects of operating temperature effects, life-time predictions, and annealing effects. The interpretation of small angle scattering is based on precipitate models which are consistant with atom probe field ion microscopy (APFIM). However, a discrepancy exists between SANS interpretation of precipitate composition on one hand and micro-chemical APFIM observation on the other. It has been observed that the magnetic-to-nuclear scattering ratio in neutron scattering is low compared to that which would be calculated with precipitate concentrations observed with APFIM. Two considerations are treated in this paper. One is the calculation of scattering from atom clusters - as distinquished from conventional precipitates - and the other is the evaluation of effects due to a lack of full magnetic saturation on the separation of nuclear and magnetic scattering effects. The calculations primarily illustrate methods for interpretation of SANS results that are not conventionally used. These calculation are limited to the simple binary iron-copper system but the methods can be easily extended to precipitate structures in pressure vessel steels.

\section{SCATTERING THEORY}

The macroscopic scattering cross section for unpolarized neutrons from copper-rich precipitates in an iron matrix in a magnetized state is written (4)

$$
\frac{d \Sigma}{d \Omega}(Q)=N_{P} V_{P}\left\{\Delta \rho_{N}^{2} F_{N}^{2}+\sin ^{2} \alpha \cdot \Delta \rho_{M}^{2} F_{M}^{2}\right\}
$$

where $Q$ is the scattering vector, $4 \pi \sin (\theta) / \lambda$, with $\theta$ the Bragg scattering angle, and $\lambda$ is the neutron wavelength, $d \Sigma / d \Omega(Q)$ is the scattering cross section in absolute units, $N_{P}$ is the number density of precipitates, $V_{P}$ is the precipitate volume, $\Delta \rho^{2} \mathrm{M}, \Delta \rho^{2} \mathrm{~N}$ are the nuclear and magnetic scattering densities, $\mathrm{F}_{\mathrm{N}}^{2}, \mathrm{~F}^{2} \mathrm{M}$, are the nuclear and magnetic scattering structure factors. The $\sin ^{2} \alpha$ factor arises from the scattering interaction between the neutron and the atomic magnetization of the scattering material,

$$
\sin ^{2} \alpha=1-(\hat{\kappa} \cdot \hat{\varepsilon})^{2}
$$

where $\kappa$ is the unit magnetization vector and $\varepsilon$ is the unit diffraction vector and $\alpha$ is the angle between the two unit vectors. The scattering densities are given by the nuclear or magnetic scattering amplitude per volume and are usually calculated on a per atom basis. The structure factor for a uniform spherical precipitate of radius $R$ is ,

"The submitted manuscript has been authored by a contractor of the U.S. Government under contract No. DE-AC05-96OR22464. Accordingly, the U.S. Government retains a nonexclusive, royalty-free license to publish or reproduce the published form of this contribution, or allow others to do so, for U.S. Government purposes."

$$
F(Q)=3(\sin Q R-Q R \cos Q R) /(Q R)^{3}
$$




\section{DISCLAIMER}

This report was prepared as an account of work sponsored by an agency of the United States Government. Neither the United States Government nor any agency thereof, nor any of their employees, makes any warranty, express or implied, or assumes any legal liability or responsibility for the accuracy, completeness, or usefulness of any information, apparatus, product, or process disclosed, or represents that its use would not infringe privately owned rights. Reference herein to any specific commercial product, process, or service by trade name, trademark, manufacturer, or otherwise does not necessarily constitute or imply its endorsement, recommendation, or favoring by the United States Government or any agency thereof. The views and opinions of authors expressed herein do not necessarily state or reflect those of the United States Government or any agency thereof. 
The scattering density contrasts for a uniform copper precipitate in iron for nuclear and magnetic scattering density respectively are $\Delta \rho_{N}=1.47 \times 10^{-12}$ and $\Delta \rho_{M}=5.07 \times 10^{-12}$. The copper precipitate is assumed to have no magnetic scattering so magnetic scattering arises from the magnetization of the iron matrix alone.

To investigate some alternative assumptions in the scattering theory, the effects of scattering from a distributed or "fuzzy" cluster and the effects of incomplete magnetic saturation in the scattering sample are examined. The structure of fuzzy cluster is considered in a recent paper on Monte Carlo sumulations of copper precipitation in dilute iron-copper alloys by Soisson et al. (5). A fuzzy cluster is taken to mean a distribution of solute with a fixed composition over an inner core which is "dressed" with a slowly decreasing density of solute on the outside. The form factor is calculated using an extension of the method used for shell-like structures found in micelle colloids. In a later section, the effects of incomplete magnetization are estimated with the use of a simple representation of domain orientations in a partially saturated magnet.

\section{Fuzzy Cluster Scattering}

If a scattering object is represented by a series of spherically symmetric shells of fixed scattering density, $\rho_{n}$, the structure factor for such a spherical micelle can be written (6),

where

$$
\begin{aligned}
F(Q)= & \left(\rho_{1}-\rho_{2}\right) V_{1} F_{0}\left(R_{1}, Q\right)+\left(\rho_{2}-\rho_{3}\right) V_{2} F_{0}\left(R_{2}, Q\right)+\cdots \\
& +\left(\rho_{N}-\rho_{0}\right) V_{N} F_{0}\left(R_{N}, Q\right)
\end{aligned}
$$

$$
F_{0}\left(R_{n}, Q\right)=4 \pi \int_{0}^{R_{n}} R^{3} \frac{(\sin Q R-Q R \cos Q R)}{(Q R)^{3}} d R
$$

The calculation is extended by the following substitution,

$$
\left(\rho_{1}-\rho_{i+1}\right)=\frac{d \rho(R)}{d R} \cdot \Delta R
$$

so that the calculation of the structure factor with a continuously varying scattering density shells can be written,

$$
F\left(Q, R_{N}\right)=4 \pi \int_{0}^{R_{N}} R^{3} \frac{d \rho(R)}{d R} \frac{(\sin Q R-Q R \cos Q R)}{(Q R)^{3}} d R
$$

The scattering density of a single cluster where it is represented by a simple polynomial,

$$
\begin{aligned}
& \rho(R)=\rho_{0} \cdot\left(a+b \frac{R}{R_{0}}+c\left(\frac{R}{R_{0}}\right)^{2}+d\left(\frac{R}{R_{0}}\right)^{3}\right) \\
& \frac{d \rho(R)}{d R}=\frac{\rho_{0}}{R_{0}}\left(b+2 c\left(\frac{R}{R_{0}}\right)+3 d\left(\frac{R}{R_{0}}\right)^{2}\right)
\end{aligned}
$$

The structure factor for the cluster is written,

$$
F\left(Q, R_{N}\right)=\frac{4 \pi \rho_{0}}{R_{0}} \int_{h_{4}}^{R_{0}} R^{3}\left(b+2 c\left(\frac{R}{R_{0}}\right)+3 d\left(\frac{R}{R_{0}}\right)^{2}\right) \frac{(\sin Q R-Q R \cos Q R)}{(Q R)^{3}} d R
$$

The integration is written

$$
\begin{aligned}
& 4 \pi \rho_{0}\left\{\cos Q R\left(24 d-2 b Q^{2}-6 Q^{2} R-12 d Q^{2} R^{2}\right)+\right. \\
& \left.\sin Q R\left(7 c Q+24 d Q R-b Q^{3} R-2 c Q^{3} R^{2}-3 d Q^{3} R^{3}\right)\right\} / Q^{6}
\end{aligned}
$$

and the integral is obtained by evaluation between the upper and lower limits of $R$.

The scattering densities in Fig 1. represent the distribution of copper with varying precipitate core radii. The maximum radius is chosen in each case to so that the core and the "dressing" region enclose the same amount of copper. 

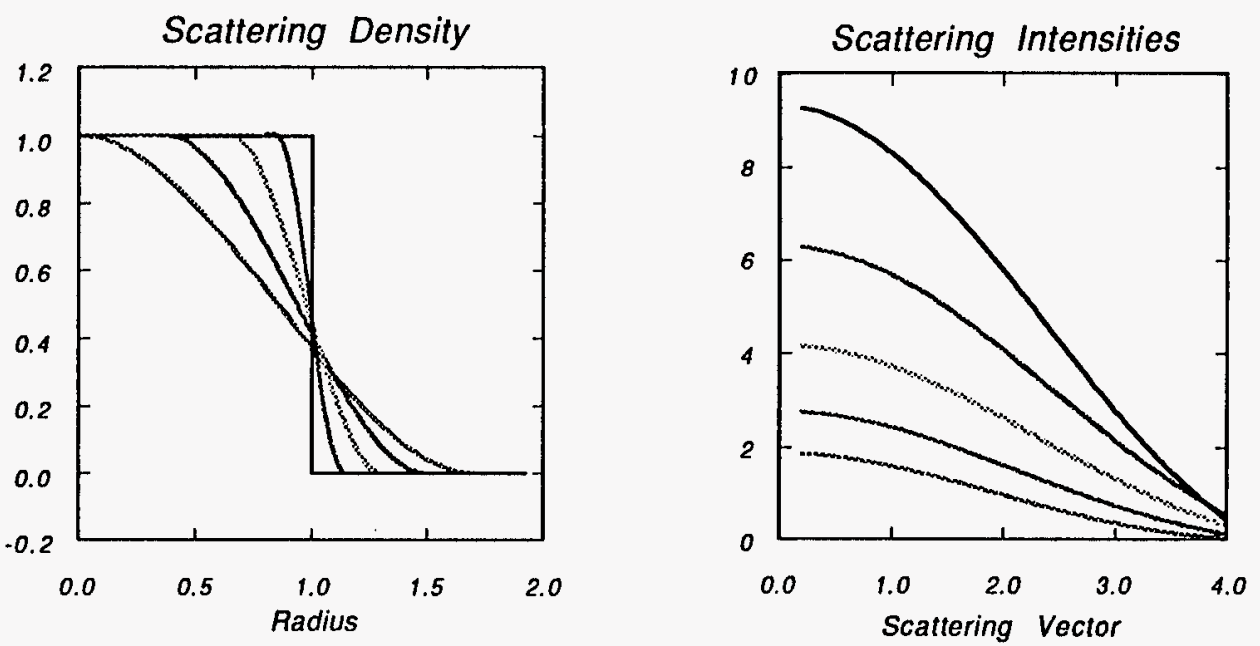

Figure. 1. The scattering densities shown on the left represent cluster structures in which the solid core is "dressed" with a continuously decreasing density of copper. The solid core radius varies from zero to $1.0 \mathrm{in}$ steps of 0.25 . The resulting scattering on the right results in a decrease in intensity as the core decreases. The result in this case does not reduce the magnetic-to-nuclear scattering ratio if it is assumed that the non-mangetic character of the cluster is unchanged.

Magnetic penetration is represented by a quadratic form for magnetic density where the magnetization extends from the iron matrix into the precipitate while decaying to zero with a quadratic dependence. Three boundary conditions are used which set the density equal to that of iron at the maximum radius and zero at the limiting penetration radius. The derivative of the density is set to zero at the maximum. Fig. 2 shows the reduction of scattering in the scattering vector range of 0 to $4 \mathrm{~nm}^{-1}$ and the maximum radius is $1 \mathrm{~nm}$. If the nuclear scattering were assumed to be fixed, then a reduction of the magnetic to nuclear scattering ratio by a factor of about two could be achieved with a penetration to a radius of $0.75 \mathrm{~nm}$.
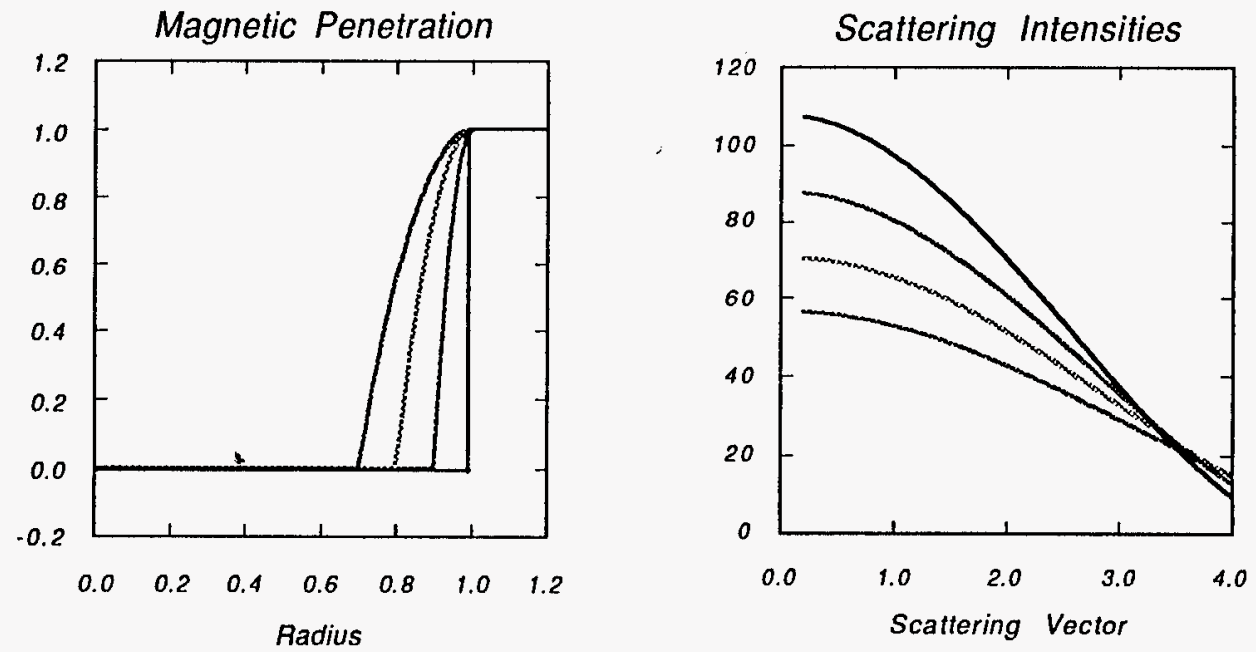

Figure 2. The magnetic scattering density profiles show varying magnetic penetrations into the precipitate. The magnetic scattering is reduced as a result of the penetration. This suggests a possible mechanism for reducing the magnetic-to-nuclear scattering ratio.

\section{Partially Saturated Magnetic Scatterer}

The scattering from each precipitate or cluster is assumed to occur within a magnetic domain. The magnetization of these domains may vary over a $4 \pi$ solid angle. The number of domains with magnetization in the range $\phi$ to $\phi+\Delta \phi$ 


$$
N(\phi) d \phi=\frac{1}{\gamma(a-\cos \phi)} d \phi \quad \text { with } \quad \gamma=\ln \frac{a+1}{a-1}
$$

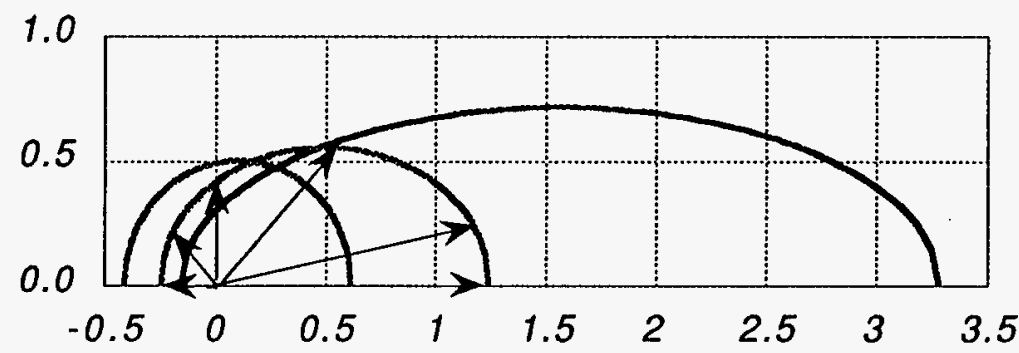

Figure 2. The magnetization distribution is shown for a-values which represent an series going from low magnetization to higher magnetization. The length of the arrow is the magnetization in the direction of the arrow.

The unit diffraction vector is $\varepsilon$ and the unit magnetization vector is $\kappa$. The magnetic interaction is given in Eq. 2 above and is evaluated for a specific diffraction vector azimuthal angle allowing the magnetization vector to vary over $4 \pi$. For an unmagnetized sample the calculation is

$$
\int_{0}^{2 \pi \pi} \int_{0}^{\pi}\left(1-(\hat{\kappa} \cdot \hat{\varepsilon})^{2}\right) \sin \phi d \phi d \eta
$$

The unit diffraction vector is written $\hat{\varepsilon}=\cos \alpha \cdot \vec{i}+\sin \alpha \cdot \vec{j}$, where the diffraction vector is taken to be in the plane of the detector with the incident beam normal to the detector plane. The unit magnetization vector is written

$$
\hat{\kappa}=\cos \phi \cdot \vec{i}+\sin \phi \cos \eta \cdot \vec{j}+\sin \phi \sin \eta \cdot \vec{k}
$$

Figure 3. The magnetic scattering variation for several magnetizations goes from a full sinusoidal variation for perfect magnetization to a constant scattering level for zero magnetization. The reduced variations still follow a $\sin ^{2} \alpha$ dependence.The magnetization values are shown in the inset.

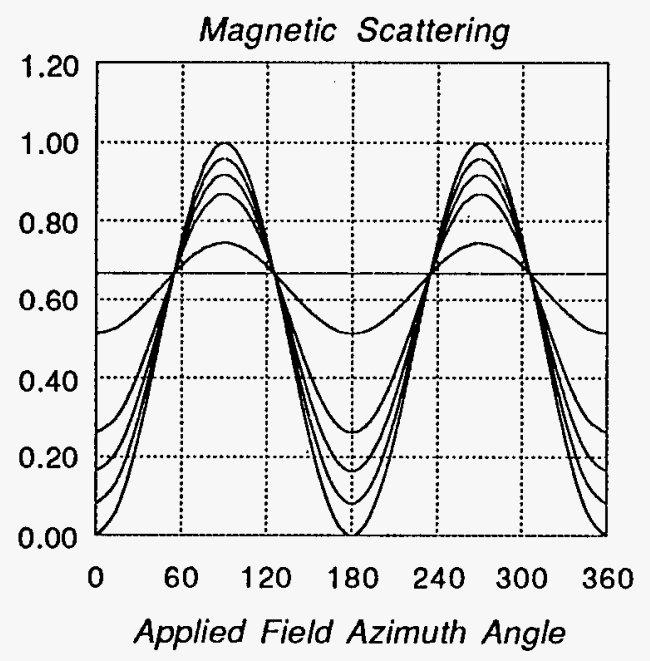

Figure 3. The magnetic scattering variation for several magnetizations goes from a full sinusoidal variation for perfect magnetization to a constant scattering level for zero magnetization. The reduced variations still follow a $\sin ^{2} \alpha$ dependence.The magnetization values are shown in the inset.

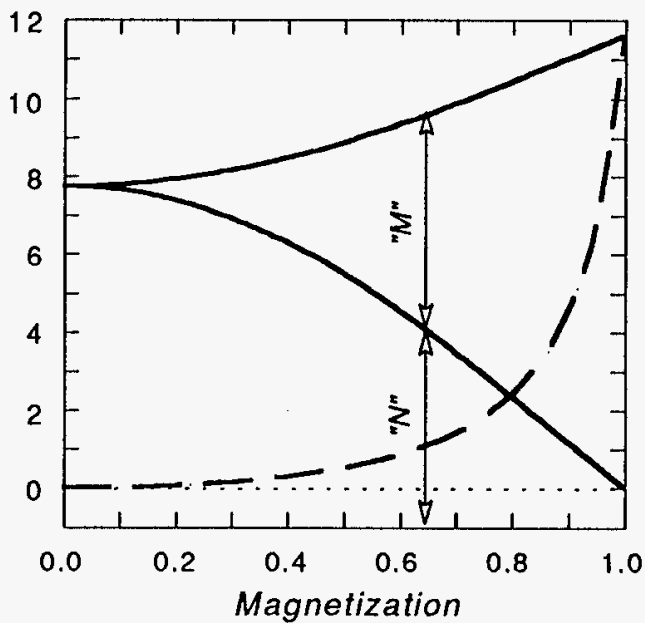

Figure 4. The maximum and minimum scattering (solid lines) is plotted as a function of the magnetization (defined in eqn. 24). The ratio of the difference between the maximum and minimum, " $M$ ", to the minimum, " $N$ ", is the apparent magnetic-to-nuclear ratio which varies strongly as magnetization approaches unity. This suggests that a very large error could be made in interpretation of the scattering if the sample were not fully saturated. 
The angle $\phi$ is measured relative to the $x$-axis and the angle $\eta$ is a rotation about the $x$-axis. Then

$$
\begin{array}{r}
(\hat{\kappa} \cdot \hat{\varepsilon})^{2}=\cos ^{2} \phi \cos ^{2} \alpha+\sin ^{2} \phi \sin ^{2} \alpha \cos ^{2} \eta+ \\
2 \cos \phi \sin \phi \cos \alpha \sin \alpha \cos \eta
\end{array}
$$

For the partially saturated case represented by the domain distribution function,

$$
\begin{array}{r}
I_{M}(a ; \alpha)=\frac{2 \pi}{\gamma} \int_{0}^{\pi} \frac{\left(1-\cos ^{2} \phi \cos ^{2} \alpha\right)}{(a-\cos \alpha)} \sin \phi d \phi- \\
\frac{\pi}{\gamma} \int_{0}^{\pi} \frac{\sin ^{2} \phi \sin ^{2} \alpha}{(a-\cos \alpha)} \sin \phi d \phi
\end{array}
$$

The magnetization, given by the average projection of magnetization along the $x$-axis, is written,

$$
M=a-\frac{2}{\gamma}
$$

Figure 3 shows the variation of the nuclear and magnetic scattering. The magnetic scattering contains a constant intensity contribution which looks like nuclear scattering. Figure 4 shows the variation of the maximum and minimum in the calculated scattering of Figure 3. The difference between the limits, labled " $\mathrm{M}$ ", goes to a limit of $\mathbf{1 1 . 6}$ for full saturation. The nuclear scattering shown at the bottom of the graph as a dotted line is 1.0. The apparent magnetic to nuclear scattering ratio is calculated from the max-min difference at a given magnetization divided by the minimum and is plotted as a dashed line. The increase in this ratio as saturation is reached is very rapid and suggests that full saturation is critical for an accurate separation of the magnetic scattering from the nuclear scattering.

\section{DISCUSSION}

A plausible model for copper precipitates combines the distributing of solute into a diffuse shell surrounding a uniform core along with a partial magnetic penetration into the cluster. The magnetization drops from that of iron at the outer radius of the cluster to zero at some distance into the cluster. Fig. 5 illustrates this case where the nuclear scattering for a fuzzy cluster with a core radius of

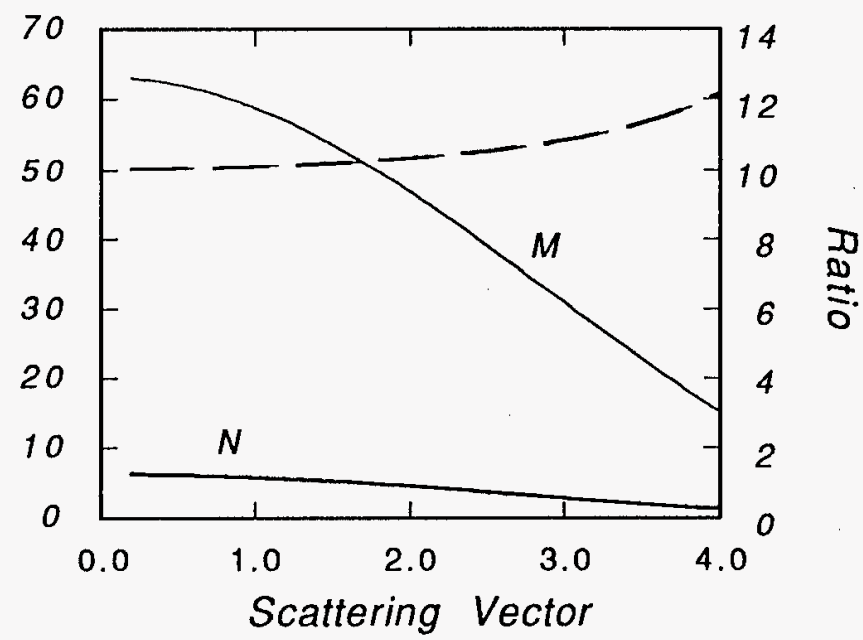

Figure 5. One example of combining the model of a fuzzy cluster, labled $N$ (core radius $=0.75$ ) and magnetic penetration, labled $M$ (penetration $=0.25$ ) results in a limited change in the scattering ratio (long dashed line). The "reduction" in the effective size of the nuclear and magnetic scattering object nearly cancels out any ratio change. Note the reduction in the intensity values. 
0.75 was combined with the magnetic scattering for magnetic penetration to a radius of 0.75 . The ratio in fact shows only a small reduction. The two intensities are significantly reduced, however.

The representation of the precipitates as a copper-rich clusters reduces the nuclear scattering intensity. And the magnetic scattering is unaltered if is assumed that the entire cluster is non-magnetic. A rmagnetic/nuclear ratio reduction would require a deep magnetic penetration in these clusters.

The magnetic domain structure based on the simple orientation distribution model in this paper demonstrates that significant errors of interpretation occur when the scattering sample is not fully saturated. This problem is amplified in the iron-copper case where the magnetic-to-nuclear ratio is very high. Such systematic errors can be avoided by first measuring the scaatering behavior as a function of field.

\section{CONCLUSIONS}

It is suggested that of the two effects discussed in this paper, the lack of magnetic saturation of the SANS scattering sample may be the more likely factor in explaining some of the differences between the APFIM and SANS interpretation. Extending the cluster scattering model to include the consideration of ternary and higher constituants with micro-segrated shells could provide a direction for refined analysis. Cluster structure obtained from microscopy or micro-chemical computations could then be used as input to the analysis and thereby improve the quantification of precipitate analysis. In extending the analysis of cluster structure, one must measure scattering over a larger range of scattering angle so that details at higher spatial resolution can be examined.

\section{ACKNOWLEDGEMENTS}

The author thanks M. Sokolov, W. Pavenich, M. Miller, P. Pareige, B. Wirth and R. Odette for very useful discussions of irradiated pressure vessel steel structures. Oak Ridge National Laboratory is managed by Lockheed Martin Energy Research Corp. under contract DE-AC05-96OR22464 for the U. S. Department of Energy.

\section{REFERENCES}

1. G. Solt, F. Frisius, and W. B. Beaven, Radiation Embrittlement of Nuclear Reactor Pressure Vessel Steels: An International Review, 3rd Vol., ASTM STP 1011, L. E. Steels, Ed.,, pp. 229-242,(1989).

2. P. A. Beaven, F. Frisius, R. Kampmann, R. Wagner, and J. R. Hawthorne, ibid., pp. 243-256.

3. G. Brauer, F. Eichorn, F. Frisius, and R. Kampmann, Effects of Radiation on Materials: 16th Intern. Symp., ASTM STP 1175, A. S. Jumar, et al., eds., pp. 503-515, (1993)

4. G. E. Bacon, Neutron Scattering, 3rd Ed., Oxford Press, New York, (1980).

5. F. Soisson, A. Barbu, and G. Martin, Acta Mater. 44, pp. 3789-3800 (1996).

6. J. B. Hayter, Fermi Summer School, Course XC, "Fisica degli anfifili: Micelle, vescicole e microemulsioni", August 1983, Soc. Ital. Fis., Bologna(1985) 


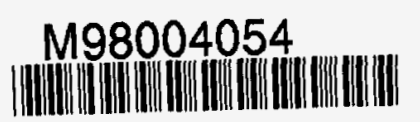

Report Number (14) ORNL/CP- -96408
CONF-96/202--

Publ. Date (11) $\frac{199711}{D O E / E R, X F}$
Sponsor Code (18)
UC Category (19) 